\title{
ON A PROPERTY OF POSITIVE-DEFINITE FUNCTIONS ${ }^{1}$
}

\author{
BY R. G. LAHA
}

Communicated by S. Bochner, May 20, 1960

In the present note we prove the following theorem:

THEOREM. Let $\left\{t_{k}\right\}(k= \pm 1, \pm 2, \cdots)$ be a sequence of real numbers such that $t_{k}>0$ and $t_{-k}=-t_{k}$ for any $k>0$ and that $t_{k} \rightarrow 0$ as $k \rightarrow \infty$. Let $f(t)$ be a positive-definite function of the real variable $t$ and let $\psi(z)$ be a function of the complex variable $z(z=t+i v, t$ and $v$ both real $)$ which is regular in a circle about the point $z=0$. Suppose that the function $f(t)$ coincides with $\psi(t)$ for every value of the sequence $\left\{t_{k}\right\}$. Then $f(t)$ coincides with $\psi(t)$ for all real $t$.

PROOF. Let the function $\psi(z)$ be regular in the circle $|z|<R(R>0)$ about the point $z=0$. Then for every real $t$ in the interval $|t|<R$ we have

$$
\psi(t)=\sum_{j=0}^{\infty}\left(\alpha_{j}+i \beta_{j}\right) \frac{t^{j}}{j !}
$$

where $\alpha_{j}$ and $\beta_{j}$ are real numbers. Let $A(t)$ and $B(t)$ denote respectively the real and imaginary parts of $\psi(t)$ so that

$$
A(t)=\sum_{j=0}^{\infty} \alpha_{j} \frac{t^{j}}{j !} \text { and } B(t)=\sum_{j=0}^{\infty} \beta_{j} \frac{t^{i}}{j !} .
$$

We now make use of the hermitian property of $f(t)$ and the equation $f\left(t_{k}\right)=\psi\left(t_{k}\right)$ and obtain easily the relations $A\left(-t_{k}\right)=A\left(t_{k}\right)$ and $B\left(-t_{k}\right)=-B\left(t_{k}\right)$ holding for every value of the sequence $\left\{t_{k}\right\}$. Since the point $t=0$ is the limit point of the sequence of real numbers $\left\{t_{k}\right\}$, we can verify easily that

$$
A(t)=\sum_{j=0}^{\infty} \alpha_{2 j} \frac{t^{2 j}}{(2 j) !} \text { and } B(t)=\sum_{j=0}^{\infty} \beta_{2 j+1} \frac{t^{2 j+1}}{(2 j+1) !}
$$

for all real $t$ in $|t|<R$.

We next introduce the functions $g(t)=f(t) f(-t)$ and $\theta(z)$ $=\psi(z) \psi(-z)(z=t+i v, t$ and $v$ real $)$ and note the following: The function $g(t)$ is a real-valued, even and positive-definite function of the real variable $t$; the function $\theta(z)$ is regular in the same circle $|z|<R$ about the point $z=0$ and for real $t$

${ }^{1}$ This work was supported by the National Science Foundation through grant NSF-G-4220 and NSF-G-9968. 


$$
\theta(t)=|\psi(t)|^{2}=\{A(t)\}^{2}+\{B(t)\}^{2}=\sum_{j=0}^{\infty} \gamma_{2 j} \frac{t^{2 j}}{(2 j) !}
$$

where $\gamma_{2 j}$ is real and therefore $\theta(t)$ is a real-valued and even function of $t$. Before proceeding further we first prove the following lemma:

LEMma. The function $g(t)$ has finite derivatives of all orders for every real $t$.

Proof of the Lemma. We can represent $g(t)$ in the form $g(t)$ $=\int_{-\infty}^{\infty} \cos t x d G(x)$ where $G(x)$ is a nondecreasing function of bounded variation. We show first that the function $g(t)$ can be differentiated twice for any real $t$. We note that the relation $g\left(t_{k}\right)=\theta\left(t_{k}\right)$ holds for every value of the sequence $\left\{t_{k}\right\}, t_{k} \rightarrow 0$ so that we must have $g(0)$ $=\theta(0)$ and therefore $\gamma_{0}=\int_{-\infty}^{\infty} d G(x)>0$. Thus for every value of the sequence $\left\{t_{k}\right\} t_{k} \rightarrow 0$ we have

$$
\frac{g\left(t_{k}\right)-g(0)}{t_{k}^{2}}=\frac{\theta\left(t_{k}\right)-\theta(0)}{t_{k}^{2}}=\frac{\gamma_{2}}{2}+O\left(t_{k}^{2}\right)
$$

so that

$$
\lim _{k \rightarrow \infty} \frac{g\left(t_{k}\right)-g(0)}{t_{k}^{2}}=\frac{\gamma_{2}}{2}
$$

exists and is finite. But we note that

$$
\frac{g\left(t_{k}\right)-g(0)}{t_{k}^{2}}=-2 \int_{-\infty}^{\infty} \frac{\sin ^{2} \frac{t_{k} x}{2}}{t_{k}^{2}} d G(x)
$$

and therefore

$$
\int_{-\infty}^{\infty} \frac{\sin ^{2} \frac{t_{k} x}{2}}{t_{k}^{2}} d G(x)
$$

tends to a finite limit as $t_{k} \rightarrow 0$. Then we use Fatou's Lemma and deduce immediately that $\int_{-\infty}^{\infty} x^{2} d G(x)<\infty$, that is, $\left|g^{\prime \prime}(0)\right|<\infty$. But it is well known that the existence of the $2 p$ th derivative of a positivedefinite function at the origin $t=0$ ensures that it is differentiable $2 p$ times for any real $t$. Therefore $g^{\prime \prime}(t)$ exists and is finite for any real $t$. We now prove the lemma by induction. We suppose that the function $g(t)$ has finite derivatives up to an even order $2 n$ and then show that the derivative of order $2 n+2$ will also exist. We note that 
the function $g(t)-\theta(t)$ is real-valued, even and can be differentiated $2 n$ times and further vanishes for every value of the sequence $\left\{t_{k}\right\}$, $t_{k} \rightarrow 0$. We now apply the Theorem of Rolle: Between two zeros of a differentiable function there exists at least one zero of its derivative. Therefore, it follows that the function $g^{\prime}(t)-\theta^{\prime}(t)$ vanishes for every value of a sequence $\left\{t_{k_{1}}\right\}$ of real numbers, $t_{k_{1}} \rightarrow 0$ which lie between the numbers of the sequence $\left\{t_{k}\right\}$. We thus apply Rolle's Theorem successively $2 n$ times and finally conclude that the function $g^{(2 n)}(t)$ coincides with the function $\theta^{(2 n)}(t)$ for every value of a sequence $\left\{t_{k_{2 n}}\right\}$ of real numbers, $t_{k_{2 n}} \rightarrow 0$. For simplicity in notation we denote this sequence by $\left\{t_{k}^{\prime}\right\}$. The relation $g^{(2 n)}\left(t_{k}^{\prime}\right)=\theta^{(2 n)}\left(t_{k}^{\prime}\right)$ holds for every value $t_{k}^{\prime}, t_{k}^{\prime} \rightarrow 0$ so that we must have $g^{(2 n)}(0)=\theta^{(2 n)}(0)$ and therefore $(-1)^{n} \gamma_{2 n}=\int_{-\infty}^{\infty} x^{2 n} d G(x)>0$. Thus for every value of the sequence $\left\{t_{k}^{\prime}\right\}, t_{k}^{\prime} \rightarrow 0$ we have

$$
\frac{g^{(2 n)}\left(t_{k}^{\prime}\right)-g^{(2 n)}(0)}{t_{k}^{\prime 2}}=\frac{\theta^{(2 n)}\left(t_{k}^{\prime}\right)-\theta^{(2 n)}(0)}{t_{k}^{\prime 2}}=\frac{\gamma_{2 n+2}}{2}+O\left(t_{k}^{\prime 2}\right)
$$

so that

$$
\lim _{x \rightarrow \infty} \frac{g^{(2 n)}\left(t_{k}^{\prime}\right)-g^{(2 n)}(0)}{t_{k}^{\prime 2}}
$$

exists and is finite. But we can verify easily that

$$
\frac{g^{(2 n)}\left(t_{k}^{\prime}\right)-g^{(2 n)}(0)}{t_{k}^{\prime 2}}=(-1)^{n+1} 2 \int_{-\infty}^{\infty} x^{2 n} \frac{\sin ^{2} \frac{t_{k}^{\prime} x}{2}}{t_{k}^{\prime 2}} d G(x)
$$

so that the integral on the right-hand side tends to a finite limit as $t_{k}^{\prime} \rightarrow 0$. We apply again Fatou's Lemma and deduce that $\int_{-\infty}^{\infty} x^{2 n+2} d G(x)$ $<\infty$ that is, $g^{(2 n+2)}(0)$ exists. Therefore $g^{(2 n+2)}(t)$ exists and is finite for any real $t$, thus completing the induction.

Now we turn to the proof of the theorem. We note that the function $g(t)$ has finite derivatives of all orders and further $g^{(2 n)}(0)$ $=\theta^{(2 n)}(0)$ for every $n$. Hence we have

$$
\limsup _{n \rightarrow \infty}\left[\frac{\left|g^{(2 n)}(0)\right|}{(2 n) !}\right]^{1 / 2 n}=\limsup _{n \rightarrow \infty}\left[\frac{\left|\theta^{(2 n)}(0)\right|}{(2 n) !}\right]^{1 / 2 n}=\frac{1}{R}
$$

so that the positive definite function $g(z)$ as a function of the complex variable $z$ is also regular in the circle $|z|<R$ about the point $z=0$. Then it follows immediately from the theorem of Raikov [3] that the positive definite function $f(z)$ is also regular in the same circle 
$|z|<R$ about the point $z=0$. Thus we conclude that both the functions $f(z)$ and $\psi(z)$ are regular in the circle $|z|<R$ and further note that they coincide for every value of a sequence $\left\{t_{k}\right\}$ of real numbers, $t_{k} \rightarrow 0$. Therefore the functions $f(z)$ and $\psi(z)$ coincide for every $z$ in the circle $|z|<R$. It then follows at once from the theorem due to Boas [1] that the function $f(t)$ coincides with $\psi(t)$ for every real $t$ and further the function $f(z)$ ( $z$ complex) is regular in the strip $|\operatorname{Im} z|<R$.

The following corollary is an immediate consequence of the above theorem:

Corollary. Let $f(t)$ be a real-valued, even and positive-definite function of the real variable $t$ and let $\psi(z)$ be a regular function of the complex variable z such that $\psi(t)$ is real-valued and even. Suppose that $f(t)$ coincides with $\psi(t)$ for every value of the sequence $\left\{t_{k}\right\}$ of real numbers, $t_{k} \rightarrow 0$. Then $f(t)$ coincides with $\psi(t)$ for every real $t$.

This result has been proved by Linnik in [2] under the additional condition that the function $\psi(t)$ is also a positive-definite function.

\section{REFERENCES}

1. R. P. Boas, Sur les series et integrales de Fourier a coefficients positifs, C. R. Acad. Sci. Paris vol. 228 (1949) pp. 1837-1838.

2. Yu. V. Linnik, The problem of determining a general probability distribution by the distribution of a statistic, (Russian), Teor. Veroyatnost. i Primenen. vol. 1 (1956) pp. 466-478.

3. D. A. Raikov, On the decomposition of Gauss and Poisson laws, (Russian), Izv. Akad. Nauk SSSR. Ser. Mat. vol. 1 (1938) pp. 91-124.

Catholic University of America 